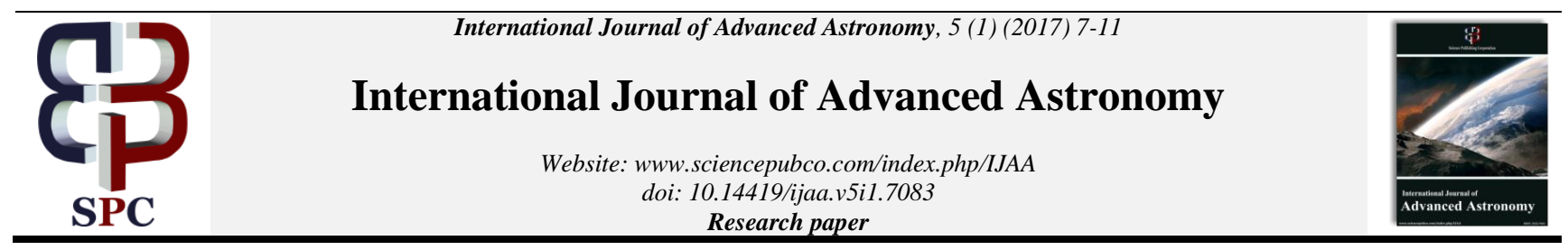

\title{
On $\mathbf{J}_{2}$ short-term orbit predictions in terms of KS elements
}

\author{
Sharon Sara Saji ${ }^{1,2}$, Harishkumar Sellamuthu ${ }^{1}$, Ram Krishan Sharma ${ }^{1 *}$ \\ ${ }^{1}$ Department of Aerospace Engineering, Karunya University, Coimbatore - 641114, Tamil Nadu, India \\ 2 Team Indus - Axiom Research Labs, Bengaluru - 560092, Karnataka, India \\ *Corresponding author E-mail: ramkrishan@karunya.edu
}

\begin{abstract}
Sharma's singularity-free analytical theory for the short-term orbital motion of satellites in terms of KS elements in closed form in eccentricity with Earth's zonal harmonic term $\mathrm{J}_{2}$, is improved by using King-Hele's expression for the radial distance ' $\mathrm{r}$ ' which includes the effect of $\mathbf{J}_{2}$, and is suitable for low eccentricity orbits. Numerical experimentation with four test cases with perigee altitude of $200 \mathrm{~km}$ and eccentricity varying from 0.01 to 0.3 for different inclinations is carried out. It is found that the orbital elements computed with the analytical expressions in a single step during half a revolution match very well with numerically integrated values and show significant improvement over the earlier theory. The solution can be effectively used for computation of mean elements for near-Earth orbits, where the short-term orbit perturbations due to $\mathrm{J}_{2}$ play most important role. The theory will be very useful in computing the state vectors during the coast phase of rocket trajectories and flight algorithms for on-board implementation.
\end{abstract}

Keywords: Analytical Integration; KS Element Equations; Earth's Oblateness; Short-Term Orbit Prediction; Radial Distance.

\section{Introduction}

The development of analytical theories for the motion of an Earth satellite under the influence of Earth's oblateness, viz. the second zonal harmonic $\mathbf{J}_{2}$, draws great attention due to the nonintegrability of the main problem in the artificial satellite theory and it may also induce chaotic motion under certain conditions [4]. Brouwer [3] and Kozai [9] made significant contributions on the study of $\mathbf{J}_{2}$ perturbed satellite motion, among several theories that emerged in the past. Lyddane [12] re-worked Brouwer's theory to improve the solution for small eccentricities and inclinations. Garfinkel [7] and Aksnes [1] employed the use of intermediary orbit to obtain a solution in terms of Delaunay's variables. Deprit's Radial Intermediary [5] is a particular canonical transformation theory of Lie type to remove the parallactic factors by the elimination of parallax, which was utilized to produce a viable alternative for the onboard orbit propagators [8].

The classical Newtonian equations, being unstable, are not very ideal choice for numerical integration as well as for development of analytical theories. Regularization of the classical Newtonian equations by Kustaanheimo and Stiefel[11] and presented in details in Stiefel and Scheifele [15], drew significant attention when $\mathrm{J}_{2}$ perturbed Lambert-type solutions in terms of $\mathrm{KS}$ (Kustaanheimo-Stiefel) transformation was derived in closed-form by Andrus [2] and Engels \& Junkins [6]. Sharma [13] obtained a singularity-free analytical solution for short-term orbit computation in terms KS elements with $J_{2}$, in series expanded up to fourth power in eccentricity. He revisited the problem and provided an accurate theory by analytical integration in closed form in eccentricity for short-term orbit prediction [14]. The equations are regular everywhere and the generalized eccentric anomaly is the independent variable. The elegant feature of KS element equations is the existence of symmetry which allows for solving only one out of the eight equations, which improves the efficiency in terms of memory usage and computation time.
In spite of the good accuracy of Sharma's closed form solution, some avenue for further improvement is available. The radial distance ' $r$ ', appearing in the denominator of the equations of motion, was assumed by Sharma to be unperturbed, i.e., $r=a(1-e \cos E)$, where a, e and $\mathrm{E}$ are semi-major, eccentricity and eccentric anomaly, respectively. However, it needs to be function of $J_{2}$. In the present study, we have modified the above theory by using KingHele's [10] expression for the radial distance ' $r$ ' which includes the effect of $J_{2}$. It is derived for low eccentricity orbits. Numerical experimentation brings out that the orbital elements computed from the new analytical theory in a single step during half a revolution show significant improvement over the earlier theory.

\section{Equations of motion}

The KS element equations in terms of four-vectors $\alpha, \beta$ with Earth's zonal harmonic term $\mathrm{J}_{2}$ are [14]:

$\frac{d \alpha_{i}}{d E}=\frac{\mu J_{2} R^{2}}{8 w^{2}}\left[\frac{1}{r^{3}}\left\{q_{0_{i}}+q_{1_{i}} \cos E+q_{2_{i}} \sin E\right\}+\frac{3}{r^{4}}\left\{g_{0_{k}}+\right.\right.$
$\left.g_{1_{k}} \cos E+g_{2_{k}} \cos ^{2} E+g_{3_{k}} \sin E+g_{4_{k}} \cos E \sin E\right\}-$
$\frac{6}{\mathrm{r}^{5}}\left\{f_{0_{i}}+f_{1_{i}} \cos E+f_{2_{i}} \cos ^{2} E+f_{3_{i}} \cos ^{3} E+f_{4_{i}} \sin E+\right.$
$\left.\left.f_{5_{i}} \sin E \cos E+f_{6_{i}} \sin E \cos ^{2} E\right\}\right]$

where

$\mathrm{q}_{0_{\mathrm{i}}}=\beta_{\mathrm{i}} \mathrm{q}_{1_{\mathrm{i}}}=-\beta_{\mathrm{i}} \mathrm{q}_{2_{\mathrm{i}}}=\alpha_{\mathrm{i}}$

where

$\mathrm{k}=\mathrm{i}+2, \mathrm{~g}_{\mathrm{j}+4}=\mathrm{g}_{\mathrm{j}}(\mathrm{j}=1,2 ; \mathrm{p}=0,1,2,3,4)$

$\mathrm{g}_{0_{\mathrm{k}}}=\mathrm{a}_{0} \mathrm{q}_{0_{\mathrm{k}}}+\mathrm{a}_{2} \mathrm{q}_{2_{\mathrm{k}}}$ 


$$
\begin{aligned}
& g_{1_{k}}=a_{1} q_{0_{k}}+a_{0} q_{1_{k}} \\
& \mathrm{~g}_{2_{\mathrm{k}}}=\mathrm{a}_{1} \mathrm{q}_{1_{\mathrm{k}}}-\mathrm{a}_{2} \mathrm{q}_{2_{\mathrm{k}}} \\
& \mathrm{g}_{3_{\mathrm{k}}}=\mathrm{a}_{2} \mathrm{q}_{0_{\mathrm{k}}}+\mathrm{a}_{0} \mathrm{q}_{2_{\mathrm{k}}} \\
& g_{4_{k}}=a_{1} q_{2_{k}}+a_{2} q_{1_{k}} \\
& f_{0_{i}}=b_{0} q_{0_{i}}+b_{3} q_{2_{i}} \\
& f_{1_{i}}=b_{1} q_{0_{i}}+b_{0} q_{1_{i}}+b_{4} q_{2_{i}} \\
& f_{2_{i}}=b_{2} q_{0_{i}}+b_{1} q_{1_{i}}-b_{3} q_{2_{i}} \\
& f_{3_{i}}=b_{2} q_{1_{i}}-b_{4} q_{2_{i}} \\
& f_{4_{\mathrm{i}}}=\mathrm{b}_{3} \mathrm{q}_{0_{\mathrm{i}}}+\mathrm{b}_{0} \mathrm{q}_{2_{\mathrm{i}}} \\
& f_{5_{i}}=b_{4} q_{0_{i}}+b_{3} q_{1_{i}}+b_{1} q_{2_{i}} \\
& f_{6_{i}}=b_{4} q_{1_{i}}+b_{2} q_{2_{i}} \\
& \mathrm{a}_{0}=\alpha_{1} \alpha_{3}+\alpha_{2} \alpha_{4}+\beta_{1} \beta_{3}+\beta_{2} \beta_{4} \\
& a_{1}=\alpha_{1} \alpha_{3}+\alpha_{2} \alpha_{4}-\beta_{1} \beta_{3}-\beta_{2} \beta_{4} \\
& \mathrm{a}_{2}=\alpha_{1} \beta_{3}+\alpha_{2} \beta_{4}+\beta_{1} \alpha_{3}+\beta_{2} \alpha_{4} \\
& \mathrm{~b}_{0}=\mathrm{a}_{0}^{2}+\mathrm{a}_{2}^{2} \mathrm{~b}_{1}=2 \mathrm{a}_{0} \mathrm{a}_{1} \\
& b_{2}=a_{1}^{2}-a_{2}^{2} b_{3}=2 a_{0} a_{2} \quad b_{4}=2 a_{1} a_{2} \\
& \mathrm{x}_{3}=\mathrm{a}_{0}+\mathrm{a}_{1} \cos \mathrm{E}+\mathrm{a}_{2} \sin \mathrm{E} \\
& \mathrm{x}_{3}{ }^{2}=\mathrm{b}_{0}+\mathrm{b}_{1} \cos \mathrm{E}+\mathrm{b}_{2} \cos ^{2} \mathrm{E}+\mathrm{b}_{3} \sin \mathrm{E}+\mathrm{b}_{4} \sin \mathrm{E} \cos \mathrm{E} \text {. (5) }
\end{aligned}
$$

If $\alpha_{\mathrm{i}}$ is changed to $\beta_{\mathrm{i}}$ in equation (1), we substitute

$\mathrm{q}_{0_{\mathrm{i}}}=\alpha_{\mathrm{i}} \quad \mathrm{q}_{1_{\mathrm{i}}}=\alpha_{\mathrm{i}} \mathrm{q}_{2_{\mathrm{i}}}=\beta_{\mathrm{i}}$

for initial conditions

\section{Expression for radial distance ' $\mathbf{r}$ ' with $\mathbf{J}_{2}$}

Restricting up to $\mathbf{J}_{2}$ term, the radial distance $\mathrm{r}$ is given by [10]:

$$
\frac{1}{\mathrm{r}}=\mathrm{L}\left[1+\mathrm{e} \cos \theta+\frac{3}{2} \mathrm{~J}_{2} \mathrm{~V}^{*}\right]
$$

where

$$
\begin{aligned}
& \mathrm{V}^{*}=\mathrm{L}^{2} \mathrm{R}^{2}\left[\frac{2-5 \sin ^{2} \mathrm{i}}{2}-\frac{\sin ^{2} \mathrm{i}}{6} \cos (2 \theta+2 \omega)\right] \\
& \mathrm{L}=\frac{1}{\mathrm{a}\left(1-\mathrm{e}^{2}\right)} \\
& (1+\mathrm{e} \cos \theta)=\frac{1-\mathrm{e}^{2}}{1-\mathrm{e} \cos \mathrm{E}},
\end{aligned}
$$

where $\theta, i$ and $\omega$ are true anomaly, inclination and argument of perigee, respectively.

The initial value of $\mathrm{V}^{*}$ is equal to $\mathrm{V}^{*}$ at $\theta$ minus $\mathrm{V}^{*}$ at $\theta=0^{\circ}$, i.e.

$$
\begin{aligned}
& V^{*}=-\frac{L^{2} R^{2}}{3}\left[\sin ^{2} i \sin ^{2} \omega-\left(\frac{z}{r}\right)^{2}\right], \\
& \left(\frac{z}{r}\right)=\sin i \sin (\theta+\omega) .
\end{aligned}
$$

$$
\begin{aligned}
& \Lambda_{0}^{00}=\mathrm{E}, \Lambda_{1}^{00}=\frac{2}{\eta^{0.5}} \tan ^{-1}\left[\left(\frac{1+\mathrm{e}}{1-\mathrm{e}}\right)^{0.5} \tan \frac{\mathrm{E}}{2}\right] \\
& \Lambda_{\mathrm{n}}^{00}=\frac{1}{(\mathrm{n}-1) \eta}\left[\frac{\mathrm{e} \sin \mathrm{E}}{\varphi^{\mathrm{n}-1}}+(2 \mathrm{n}-3) \Lambda_{\mathrm{n}-1}^{00}-(\mathrm{n}-2) \Lambda_{\mathrm{n}-2}^{00}\right] \mathrm{n}>1 \\
& \Lambda_{\mathrm{n}}^{01}=-\frac{1}{(\mathrm{n}-1) \mathrm{e} \Lambda^{\mathrm{n}-1}} \mathrm{n}>1 \\
& \Lambda_{\mathrm{n}}^{11}=\frac{1}{\mathrm{e}}\left(\Lambda_{\mathrm{n}}^{01}-\Lambda_{\mathrm{n}-1}^{01}\right) \mathrm{n}>2
\end{aligned}
$$

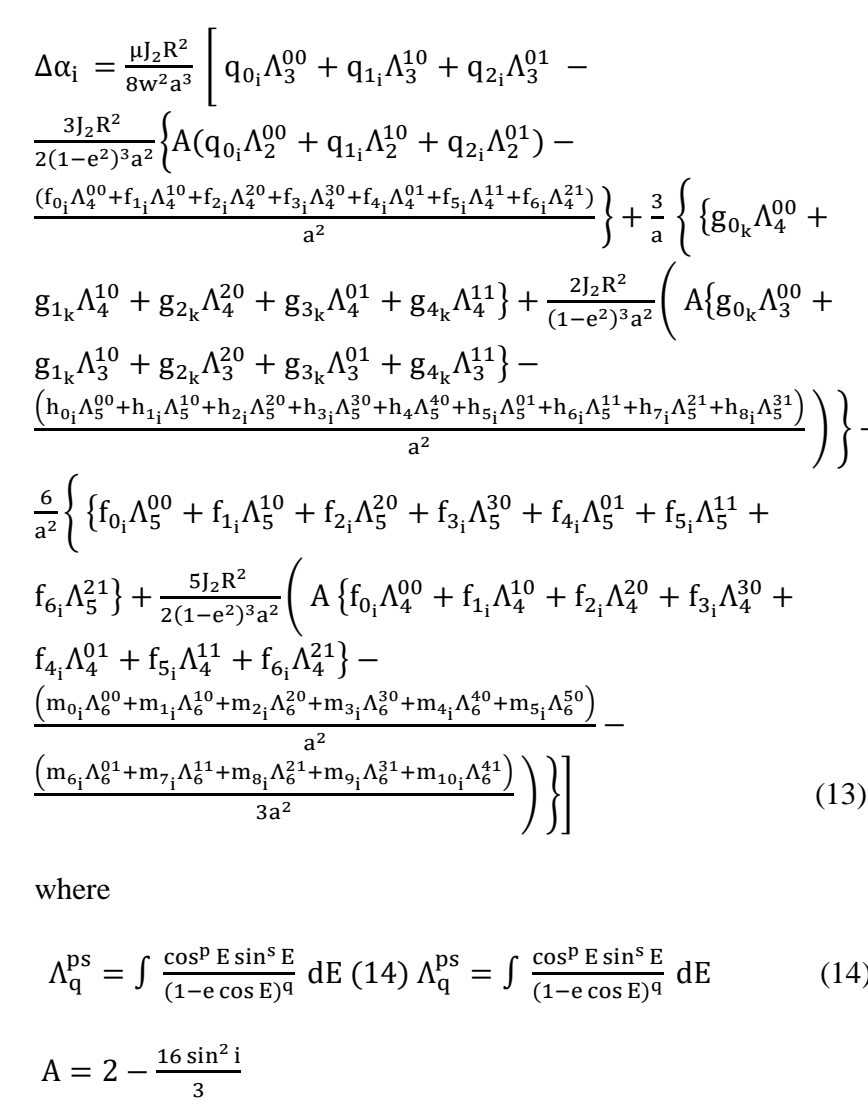
we get

$\frac{1}{\mathrm{r}^{3}}=\mathrm{L}^{3}(1+\mathrm{e} \cos \theta)^{3}\left[1-\frac{3 \mathrm{~L}_{2} \mathrm{R}^{2}}{2\left(1-\mathrm{e}^{2}\right)}(1-\mathrm{e} \cos \mathrm{E})\left\{\sin ^{2} \mathrm{i} \sin ^{2} \omega-\right.\right.$

$$
\begin{aligned}
& \frac{1}{\mathrm{r}^{4}}=\mathrm{L}^{4}(1+\mathrm{e} \cos \theta)^{4}\left[1-\frac{2 \mathrm{~J}_{2} \mathrm{~L}^{2} \mathrm{R}^{2}}{\left(1-\mathrm{e}^{2}\right)}(1-\mathrm{e} \cos \mathrm{E})\left\{\sin ^{2} \mathrm{i} \sin ^{2} \omega-\right.\right. \\
& \left.\left.\left(\frac{\mathrm{z}}{\mathrm{r}}\right)^{2}\right\}\right]
\end{aligned}
$$

(3) $\frac{1}{\mathrm{r}^{5}}=\mathrm{L}^{5}(1+\mathrm{e} \cos \theta)^{5}\left[1-\frac{5 \mathrm{~J}_{2} \mathrm{~L}^{2} \mathrm{R}^{2}}{2\left(1-\mathrm{e}^{2}\right)}(1-\mathrm{e} \cos \mathrm{E})\left\{\sin ^{2} \mathrm{i} \sin ^{2} \omega-\right.\right.$

$$
\left.\left.\left(\frac{z}{r}\right)^{2}\right\}\right]
$$

Substituting Equations (10), (11) and (12) in Equation (1) and ely, we get

$\Lambda_{5}^{21}=\frac{1}{\mathrm{e}^{2}}\left[\Lambda_{5}^{01}-2 \Lambda_{4}^{01}+\Lambda_{3}^{01}\right]$

$\Lambda_{\mathrm{n}}^{\mathrm{m} 0}=1 /(-\mathrm{e})^{\mathrm{m}} \sum_{\mathrm{k}=0}^{\mathrm{m}}\left(\begin{array}{c}\mathrm{m} \\ \mathrm{k}\end{array}\right)(-1)^{\mathrm{m}-\mathrm{k}} \Lambda_{\mathrm{n}-\mathrm{k}}^{00}$

$\Lambda_{5}^{31}=\frac{\frac{1}{1-\mathrm{e} \cos \mathrm{E}}-\frac{1.5}{(1-\mathrm{e} \cos \mathrm{E})^{2}}+\frac{1}{(1-\mathrm{e} \cos \mathrm{E})^{3}}-\frac{0.25}{(1-\mathrm{e} \cos \mathrm{E})^{4}}}{\mathrm{e}^{4}}$ 


$$
\begin{aligned}
& \Lambda_{6}^{21}=\frac{-\frac{0.2}{(1-\mathrm{e} \cos \mathrm{E})^{5}}+\frac{0.5}{(1-\mathrm{e} \cos \mathrm{E})^{4}}-\frac{1}{3(1-\mathrm{e} \cos \mathrm{E})^{3}}}{\mathrm{e}^{3}} \\
& \Lambda_{6}^{31}=\frac{\frac{-0.2}{(1-e \cos E)^{5}}+\frac{3}{4(1-e \cos E)^{4}}-\frac{1}{(1-e \cos E)^{3}}+\frac{0.5}{(1-e \cos E)^{2}}}{e^{4}} \\
& \Lambda_{6}^{41}=-\frac{\cos ^{5} \mathrm{E}}{5}-\mathrm{e} \cos ^{6} \mathrm{E}-3 \mathrm{e}^{2} \cos ^{7} \mathrm{E}-7 \mathrm{e}^{3} \cos ^{8} \mathrm{E} \\
& \text { with } \\
& \varphi=\frac{\mathrm{r}}{\mathrm{a}} \eta=1-\mathrm{e}^{2} \\
& \mathrm{~L}=\frac{1}{\mathrm{a}\left(1-\mathrm{e}^{2}\right)} \\
& (1+e \cos \theta)=\frac{1-e^{2}}{1-e \cos E} \\
& \mathrm{~h}_{0_{\mathrm{k}}}=\mathrm{b}_{3} \mathrm{~g}_{3_{\mathrm{k}}}+\mathrm{b}_{0} \mathrm{~g}_{0_{\mathrm{k}}} \\
& \mathrm{h}_{1_{\mathrm{k}}}=\mathrm{b}_{3} \mathrm{~g}_{4_{\mathrm{k}}}+\mathrm{b}_{4} \mathrm{~g}_{3_{\mathrm{k}}}+\mathrm{b}_{0} \mathrm{~g}_{1_{\mathrm{k}}}+\mathrm{b}_{1} \mathrm{~g}_{0_{\mathrm{k}}} \\
& \mathrm{h}_{2_{\mathrm{k}}}=\mathrm{b}_{4} \mathrm{~g}_{4_{\mathrm{k}}}-\mathrm{b}_{3} \mathrm{~g}_{3_{\mathrm{k}}}+\mathrm{b}_{0} \mathrm{~g}_{2_{\mathrm{k}}}+\mathrm{b}_{1} \mathrm{~g}_{1_{\mathrm{k}}}+\mathrm{b}_{2} \mathrm{~g}_{0_{\mathrm{k}}} \\
& h_{3_{k}}=-b_{3} g_{4_{k}}-b_{4} g_{3_{k}}+b_{1} g_{2_{k}}+b_{2} g_{1_{k}} \\
& \mathrm{~h}_{4_{\mathrm{k}}}=\mathrm{b}_{2} \mathrm{~g}_{2_{\mathrm{k}}}-\mathrm{b}_{4} \mathrm{~g}_{4_{\mathrm{k}}} \\
& \mathrm{h}_{5_{\mathrm{k}}}=\mathrm{b}_{0} \mathrm{~g}_{3_{\mathrm{k}}}+\mathrm{b}_{3} \mathrm{~g}_{0_{\mathrm{k}}} \\
& \mathrm{h}_{6_{\mathrm{k}}}=\mathrm{b}_{0} \mathrm{~g}_{4_{\mathrm{k}}}+\mathrm{b}_{1} \mathrm{~g}_{3_{\mathrm{k}}}+\mathrm{b}_{3} \mathrm{~g}_{1_{\mathrm{k}}}+\mathrm{b}_{4} \mathrm{~g}_{0_{\mathrm{k}}} \\
& \mathrm{h}_{7_{\mathrm{k}}}=\mathrm{b}_{1} \mathrm{~g}_{4_{\mathrm{k}}}+\mathrm{b}_{2} \mathrm{~g}_{3_{\mathrm{k}}}+\mathrm{b}_{3} \mathrm{~g}_{2_{\mathrm{k}}}+\mathrm{b}_{4} \mathrm{~g}_{1_{\mathrm{k}}} \\
& \mathrm{h}_{8_{\mathrm{k}}}=\mathrm{b}_{2} \mathrm{~g}_{4_{\mathrm{k}}}+\mathrm{b}_{4} \mathrm{~g}_{2_{\mathrm{k}}} \\
& \mathrm{m}_{0_{\mathrm{i}}}=\mathrm{b}_{0} \mathrm{f}_{0_{\mathrm{i}}}+\mathrm{b}_{3} \mathrm{f}_{4_{\mathrm{i}}} \\
& \mathrm{m}_{1_{\mathrm{i}}}=\mathrm{b}_{1} \mathrm{f}_{0_{\mathrm{i}}}+\mathrm{b}_{3} \mathrm{f}_{5_{\mathrm{i}}}+\mathrm{b}_{4} \mathrm{f}_{4_{\mathrm{i}}}+\mathrm{b}_{0} \mathrm{f}_{1_{\mathrm{i}}} \\
& \mathrm{m}_{2_{\mathrm{i}}}=\mathrm{b}_{2} \mathrm{f}_{0_{\mathrm{i}}}+\mathrm{b}_{3} \mathrm{f}_{6_{\mathrm{i}}}+\mathrm{b}_{4} \mathrm{f}_{5_{\mathrm{i}}}-\mathrm{b}_{3} \mathrm{f}_{4_{\mathrm{i}}}+\mathrm{b}_{2} \mathrm{f}_{0_{\mathrm{i}}}+\mathrm{b}_{1} \mathrm{f}_{1_{\mathrm{i}}} \\
& m_{2_{i}}=b_{2} f_{0_{i}}+b_{3} f_{6_{i}}+b_{4} f_{5_{i}}-b_{3} f_{4_{i}}+b_{2} f_{0_{i}}+b_{1} f_{1_{i}}
\end{aligned}
$$

$$
\begin{aligned}
& \mathrm{m}_{3_{\mathrm{i}}}=\mathrm{b}_{4} \mathrm{f}_{6_{\mathrm{i}}}-\mathrm{b}_{3} \mathrm{f}_{5_{\mathrm{i}}}-\mathrm{b}_{4} \mathrm{f}_{4_{\mathrm{i}}}+\mathrm{b}_{0} \mathrm{f}_{3_{\mathrm{i}}}+\mathrm{b}_{1} \mathrm{f}_{\mathrm{2}_{\mathrm{i}}}+\mathrm{b}_{2} \mathrm{f}_{1_{\mathrm{i}}} \\
& \mathrm{m}_{4_{\mathrm{i}}}=-\mathrm{b}_{3} \mathrm{f}_{6_{\mathrm{i}}}-\mathrm{b}_{4} \mathrm{f}_{5_{\mathrm{i}}}+\mathrm{b}_{1} \mathrm{f}_{3_{\mathrm{i}}}+\mathrm{b}_{2} \mathrm{f}_{2_{\mathrm{i}}} \\
& \mathrm{m}_{5_{\mathrm{i}}}=\mathrm{b}_{2} \mathrm{f}_{3_{\mathrm{i}}}-\mathrm{b}_{4} \mathrm{f}_{6_{\mathrm{i}}} \\
& \mathrm{m}_{6_{\mathrm{i}}}=\mathrm{b}_{3} \mathrm{f}_{0_{\mathrm{i}}}-\mathrm{b}_{0} \mathrm{f}_{4_{\mathrm{i}}} \\
& \mathrm{m}_{7_{\mathrm{i}}}=\mathrm{b}_{4} \mathrm{f}_{0_{\mathrm{i}}}+\mathrm{b}_{0} \mathrm{f}_{5_{\mathrm{i}}}+\mathrm{b}_{1} \mathrm{f}_{4_{\mathrm{i}}}+\mathrm{b}_{3} \mathrm{f}_{1_{\mathrm{i}}} \\
& \mathrm{m}_{8_{\mathrm{i}}}=\mathrm{b}_{0} \mathrm{f}_{6_{\mathrm{i}}}+\mathrm{b}_{1} \mathrm{f}_{5_{\mathrm{i}}}+\mathrm{b}_{2} \mathrm{f}_{4_{\mathrm{i}}}+\mathrm{b}_{3} \mathrm{f}_{2_{\mathrm{i}}}+\mathrm{b}_{4} \mathrm{f}_{\mathrm{1}_{\mathrm{i}}} \\
& \mathrm{m}_{\mathrm{g}_{\mathrm{i}}}=\mathrm{b}_{1} \mathrm{f}_{6_{\mathrm{i}}}+\mathrm{b}_{2} \mathrm{f}_{5_{\mathrm{i}}}+\mathrm{b}_{3} \mathrm{f}_{3_{\mathrm{i}}}+\mathrm{b}_{4} \mathrm{f}_{\mathrm{2}_{\mathrm{i}}} \\
& \mathrm{m}_{10_{\mathrm{i}}}=\mathrm{b}_{2} \mathrm{f}_{6_{\mathrm{i}}}+\mathrm{b}_{4} \mathrm{f}_{3_{\mathrm{i}}} .
\end{aligned}
$$

\begin{tabular}{|c|c|c|c|c|c|c|c|c|}
\hline \multirow[b]{2}{*}{ Parameter } & \multirow[b]{2}{*}{ Case } & \multirow{2}{*}{ Method } & \multicolumn{5}{|c|}{ ANAL steps (deg) } & \multirow[b]{2}{*}{180} \\
\hline & & & 30 & 60 & 90 & 150 & 165 & \\
\hline \multirow{8}{*}{$\mathrm{a}(\mathrm{m})$} & \multirow{2}{*}{ A } & NUM-ANAL1 & -0.05 & -3.6 & -20.1 & -3.8 & 21.9 & 43.8 \\
\hline & & NUM-ANAL2 & -1.33 & -14.9 & -39.3 & -3.4 & 22.9 & 44.8 \\
\hline & \multirow[b]{2}{*}{ B } & NUM-ANAL1 & -0.6 & -8.6 & -23.7 & 10.2 & 27.5 & 40.5 \\
\hline & & NUM-ANAL2 & -2.2 & -19.2 & -35.1 & 15.5 & 33.2 & 46.3 \\
\hline & \multirow{2}{*}{$\mathrm{C}$} & NUM-ANAL1 & -1.2 & -14.0 & -21.4 & 22.4 & 33.4 & 40.9 \\
\hline & & NUM-ANAL2 & -3.9 & -24.4 & -26.4 & 29.9 & 40.8 & 48.2 \\
\hline & \multirow{2}{*}{ D } & NUM-ANAL1 & 0.8 & -15.2 & -8.6 & 36.7 & 43.5 & 47.9 \\
\hline & & NUM-ANAL2 & -7.3 & -29.2 & -12.3 & 41.7 & 47.9 & 51.9 \\
\hline
\end{tabular}

\section{Numerical results}

To compute $\boldsymbol{\alpha}$ and $\boldsymbol{\beta}$, we have programed Equation (13) in double precision arithmetic on a NV57H26u Gateway computer. Four test cases A, B, C and D with eccentricities $0.01,0.1,0.2$ and 0.3 at high inclination of $85^{\circ}$ having a perigee height of $200 \mathrm{~km}$ are chosen for detailed numerical study. The initial values chosen for right ascension of ascending node $(\Omega)=60^{\circ}$ and argument of perigee $(\omega)=0^{\circ}$. Numerical integration of the KS element equations of motion with $\mathbf{J}_{2}$ [14] is carried out with fixed step-size fourthorder Runge-Kutta-Gill method with a step-size of one degree in eccentric anomaly. The difference between the numerically integrated and analytically computed values with the modified theory (ANAL1) and existing theory (ANAL2) during half a revolution with a single analytical step size are given in Table 1 . It may be noted that the modified theory provides more accurate values of the semi-major axis during most of the part of the orbit in half a revolution. The improvement in the semi-major axis with the new theory at $90^{\circ}$ step size is found to be $19.2 \mathrm{~m}, 11.4 \mathrm{~m}, 5 \mathrm{~m}$ and 3.7 $\mathrm{m}$, respectively for the four test cases, against the error of $20.1 \mathrm{~m}$, $23.7 \mathrm{~m}, 21.4 \mathrm{~m}$ and $8.6 \mathrm{~m}$, respectively. The difference between the new theory and the old theory for $\mathrm{i}=85$ degrees in semi-major axis, eccentricity and inclination are given in Figures 1,2 and 3, respectively.

Table 1: Variation in Semi-Major Axis during Half a Revolution with $\mathrm{J}_{2}$ 


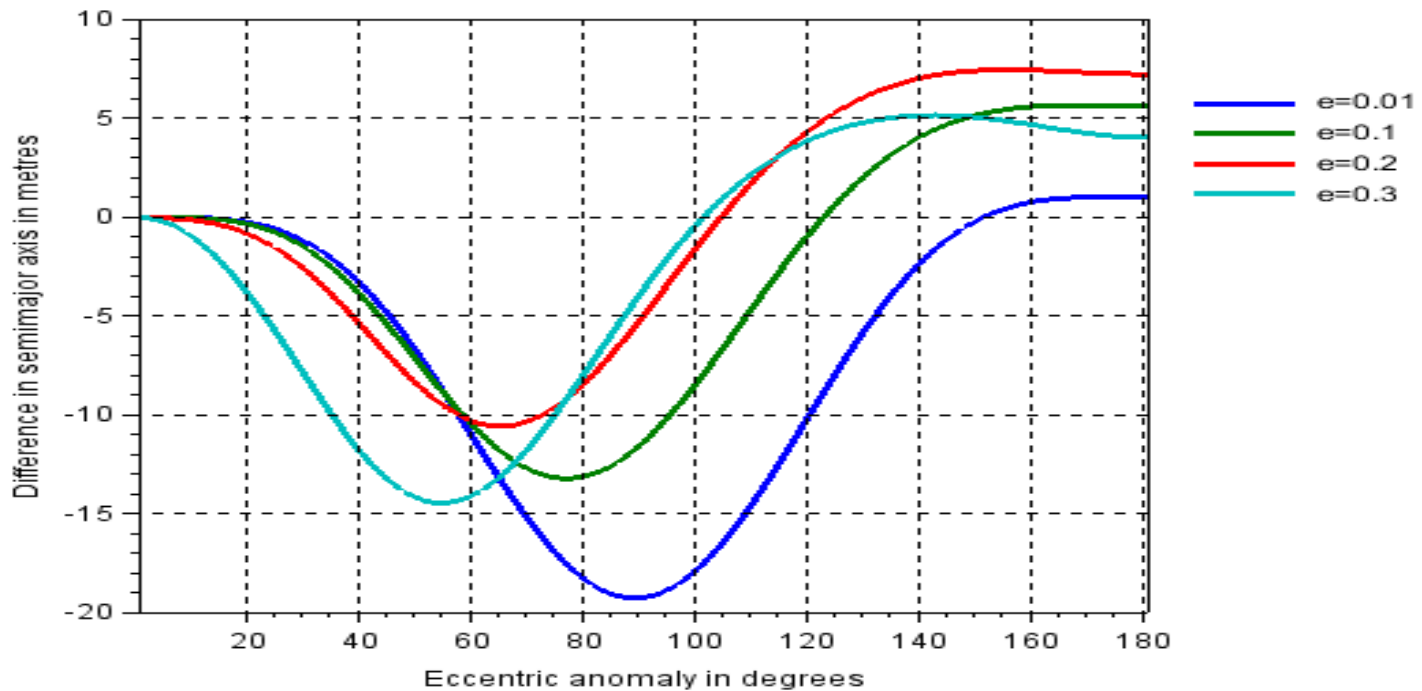

Fig. 1: Difference in Semi-Major Axis between the Modified and Existing Theory.

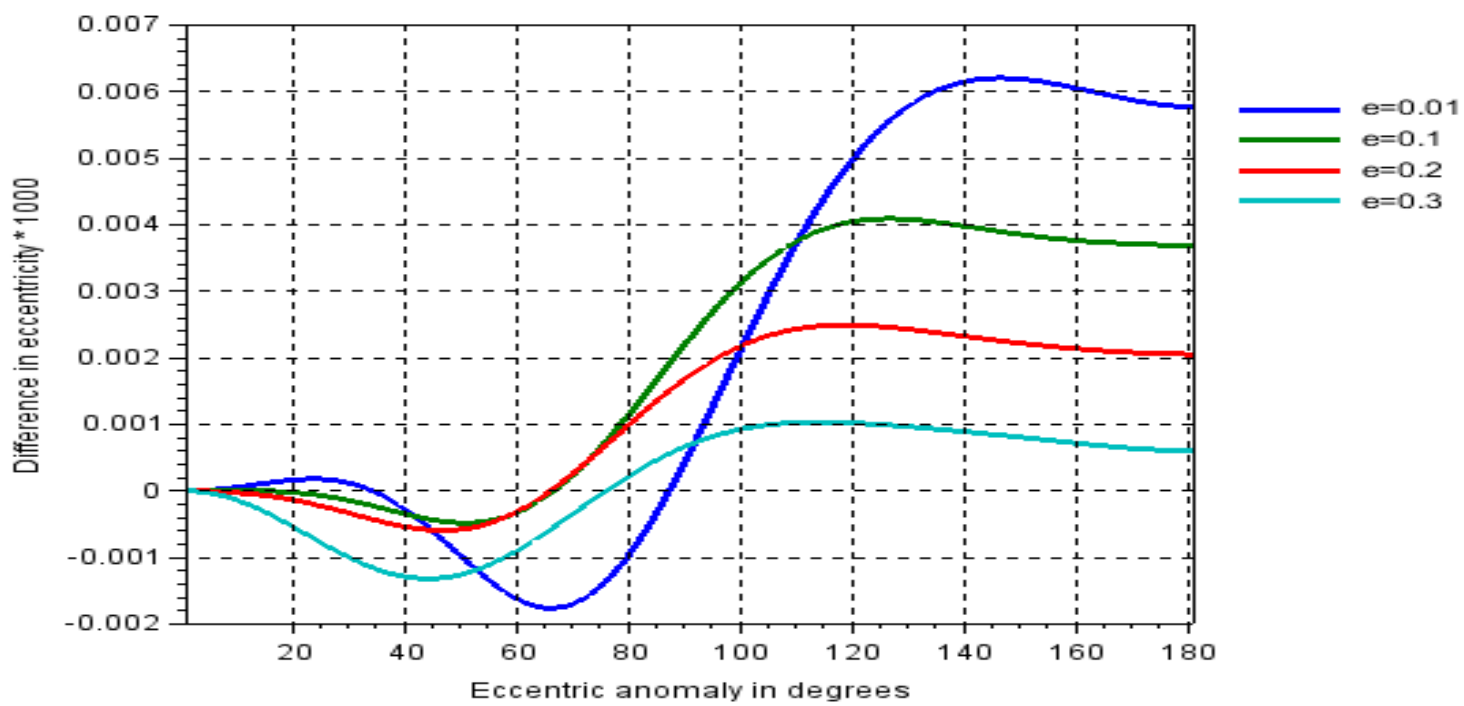

Fig. 2: Difference in Eccentricity between the Modified and Existing Theory.

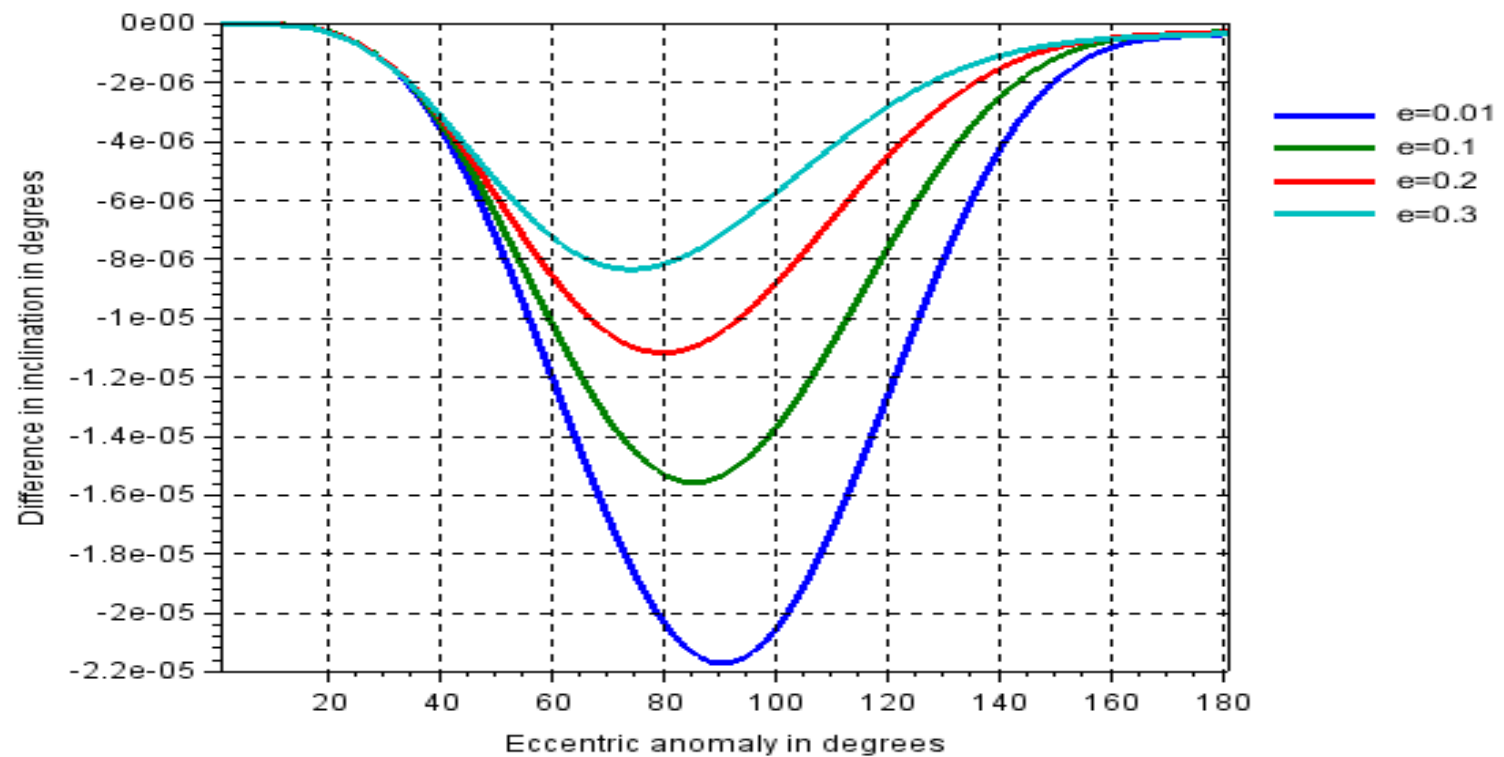

Fig. 3: Difference in Inclination between the Modified and Existing Theory.

Figures 1-3 provide the differences in semi-major axis, eccentricity and orbital inclination, respectively, between the modified and the old theory. It may be noted that the maximum improvement with the new theory is for the small eccentricity (0.01) case $\mathrm{A}$ and the magnitude of improvement in semi-major axis, eccentricity and inclination are $\sim 19 \mathrm{~m}, 6.2 \times 10^{-6}$ and $2.19^{\circ} \mathrm{x}$ $10^{-5}$, respectively. The maximum improvement happened with a single step-size in eccentric anomaly of $90^{\circ}$ for semi-major axis and inclination, and a single step-size of $\sim 148^{\circ}$ for eccentricity. 


\section{Conclusion}

A modified non-singular theory for analytical solution of shortterm motion of satellites with $\mathbf{J}_{2}$ is evolved using KS regular elements. The study showed that with the inclusion of the perturbed ' $r$ ' from King-Hele [10], the accuracy of the solution increases significantly. The results are documented up to half a revolution. The theory provides accurate solution for small as well as high inclination orbits.

\section{Acknowledgement}

The second and third authors gratefully acknowledge the support received by grant: "SR/S4/MS: 801/12" from Department of Science and Technology - Science and Engineering Research Board (DST-SERB), India to carry out this research work.

\section{References}

[1] Aksnes, K. (1970) A second-order artificial satellite theory based on an intermediate orbit. Astron. J. 75, 1066-1076. https://doi.org/10.1086/111061.

[2] Andrus, J.F. (1977) First-order effects of the earth's oblateness upon coasting bodies, Celest. Mech. 15, 217-224. https://doi.org/10.1007/BF01228463.

[3] Brouwer, D. (1959) Solution of the problem of artificial satellite theory without drag. Astron. J. 64, 378-396 https://doi.org/10.1086/107958.

[4] Celleti, A., Negrini, P. (1995) Non-integrability of the problem of motion around an oblate planet. Celest. Mech. 61, 253-260. https://doi.org/10.1007/BF00051896.

[5] Deprit, A. (1981) The elimination of the parallax in satellite theory, Celest. Mech. 24, 111-153. https://doi.org/10.1007/BF01229192.

[6] Engels, R. C. and Junkins, J. L. (1981) The gravity-perturbed Lambert problem - A KS variation of parameters approach. Celest. Mech. 24 3-21. https://doi.org/10.1007/BF01228790.

[7] Garfinkel, B. (1959) The orbits of a satellite of an oblate planet Astron. J. 64, 353. https://doi.org/10.1086/107956.

[8] Gurfil, P. and Lara, M. (2014) Satellite onboard orbit propagation using Deprit's radial intermediary. Celest. Mech. Dyn. Astron. 120, 217-232. https://doi.org/10.1007/s10569-014-9576-1.

[9] Kozai, Y. (1962) Second order solution of artificial satellite theory without air drag. Astron. J. 67, 446-461. https://doi.org/10.1086/108753.

[10] King-Hele, D. G. (1958) The effect of the Earth's oblateness on the orbit of a near satellite, Proc. R. Soc. London a 247, 49-70. https://doi.org/10.1098/rspa.1958.0169.

[11] Kustaanheimo, P. and Stiefel, E. (1965) Perturbation theory of Kepler motion based on spinor regularization, J. Reine Angew Math. 218, 204-219.

[12] Lyddane, R.H. (1963) Small eccentricities or inclinations in the Brouwer theory of the artificial satellite, Astron. J. 68, 555-558. https://doi.org/10.1086/109179.

[13] Sharma, R. K. (1989) Analytical approach using KS elements to short-term orbit predictions including $\mathrm{J}_{2}$, Celest. Mech. Dyn. Astron. 46, 321-333. https://doi.org/10.1007/BF00051486.

[14] Sharma, R. K. (1997) Analytical integration of K-S element equations with $\mathrm{J}_{2}$ for short-term orbit predictions, Planet. Space Sci. 45 1481-1486. https://doi.org/10.1016/S0032-0633(97)00093-7.

[15] Stiefel, E.L. and Scheifele, G. (1971) Linear and Regular Celestial Mechanics, Springer, Berlin. https://doi.org/10.1007/978-3-64265027-7. 